\title{
Binuclear ruthenium(II) complexes for amyloid fibrils recognition
}

\author{
Piotr Hanczyc* \\ University of California, Santa Barbara, Center for Polymers E Organic Solids, 2520A Physical Sciences Building North, Santa Barbara, United States \\ Chalmers University of Technology, Department of Chemical and Biological Engineering, Kemivagen 10, Gothenburg, 41296, Sweden
}

\section{A R T I C L E I N F O}

\section{Article history:}

Received 16 September 2014

In final form 13 October 2014

Available online 23 October 2014

\section{Keywords:}

Insulin fibrils

Ruthenium(II) complexes

Amyloid-chromophore adducts

Two-photon excited emission

Linear dichroism spectroscopy

\begin{abstract}
A B S T R A C T
Metal-organic compounds represent a unique class of biomarkers with promising photophysical properties useful for imaging. Here interactions of insulin fibrils with two binuclear complexes $\left[\mu-\left(11,11^{\prime} \text {-bidppz)(phen }\right)_{4} \mathrm{Ru}_{2}\right]^{4+}(\mathbf{1})$ and $\left[\mu-\mathrm{C} 4(\mathrm{cpdppz})(\mathrm{phen})_{4} \mathrm{Ru}_{2}\right]^{4+}(\mathbf{2})$ are studied by linear dichroism (LD) and fluorescence. These ruthenium(II) compounds could provide a new generation of amyloid binding chromophores with long lived lifetimes, good luminescence quantum yields for the bound molecules and photo-stability useful in multiphoton luminescence imaging.
\end{abstract}

() 2014 Elsevier B.V. All rights reserved.

\section{Introduction}

Fibrilization of peptides leads to formation of amyloid fibril structures [1]. The process is promoted by certain mutations that affect protein folding which may result in erratic structures, such as self-assembled isolable aggregates believed to lead to amyloidosis and in consequence to serious diseases [2-4]. One of the most common detection method for amyloids in vivo and in vitro is staining with organic dyes such as Thioflavine T and Congo Red [5]. However, those chromophores are far from being ideal, and in respect of progress in diagnostic technology, new chromophores, more effective, specific and with good photophysical properties are in demand for fibrils recognition. Transition metal complexes constitute an interesting class of probes exhibiting versatile properties that, due to the rigid coordination framework with possibilities to vary the three-dimensional structure could be tuned for specific tasks $[6,7]$. Ruthenium(II) complexes, in particular those comprising the dipyrido[3,2-a:2',3'-c]phenazine (dppz) ligand and derivatives thereof, have been intensively studied due to their environmental sensitive luminescence which make them versatile spectroscopic probes for biomolecules including amyloid fibrils [8]. Strongly hydrophobic environment leads to the efficient fluorescence, whereas polarity leads to complete quenching (so called the lightswitch effect [9]). Ruthenium complexes structure can be also extended to dimers. Monomeric $\left[\mathrm{Ru}(\mathrm{phen})_{2} \mathrm{dppz}\right]^{2+}$ moieties are connected either by a single bond between the dppz moieties that

\footnotetext{
* Address: Chalmers University of Technology, Department of Chemical and Biological Engineering, Kemivagen 10, Gothenburg, 41296, Sweden.

E-mail address: piotr.hanczyc@chalmers.se
}

is resulting in dimeric complex (1) which is relatively rigid or by connecting with aliphatic chain that makes it flexible (2) [structures: see insets in Fig. 1].

Intramolecular structure is particularly important for potential applications of these compounds for non-bleaching multiphoton imaging [10] which is using low energy photons outside the absorption edges that allows performing non-invasive in vivo studies in the near-infrared (NIR) without damaging biological materials. Moreover this detection approach has better spatial resolution and long penetration depth [11]. Thus interactions between amyloids and two binuclear ruthenium(II) complexes of different rigidity were investigated. Amyloid fibrils formed by insulin can be oriented in a flow linear dichroism (LD) [12] what opens the possibility to understand the mechanism of interactions between chromophores and the fibrils. Results from LD and fluorescence are analyzed in terms of binding geometry and accessibility for multiphoton detection.

\section{Materials and methods}

Samples were prepared as follows: a native insulin protein from bovine albumin was purchased from Sigma Aldrich, dissolved in $\mathrm{pH}=2(0.01 \mathrm{M} \mathrm{HCl})$ water buffer and adjusted to final concentration of $5 \mathrm{mg} / \mathrm{ml}$. The solution was filtered through $0.2 \mu \mathrm{m}$ filter and further heated to $65{ }^{\circ} \mathrm{C}$ for $24 \mathrm{~h}$. After fibrilization the samples were centrifuged at $3000 \mathrm{rpm}$ for $5 \mathrm{~min}$ in order to remove globular particulates. Ruthenium(II) complexes were kindly provided by Per Lincoln at Chalmers University of Technology.

Linear dichroism spectra of insulin fibrils alone and in mixture with ruthenium(II) complexes were recorded on a Chirascan CD 

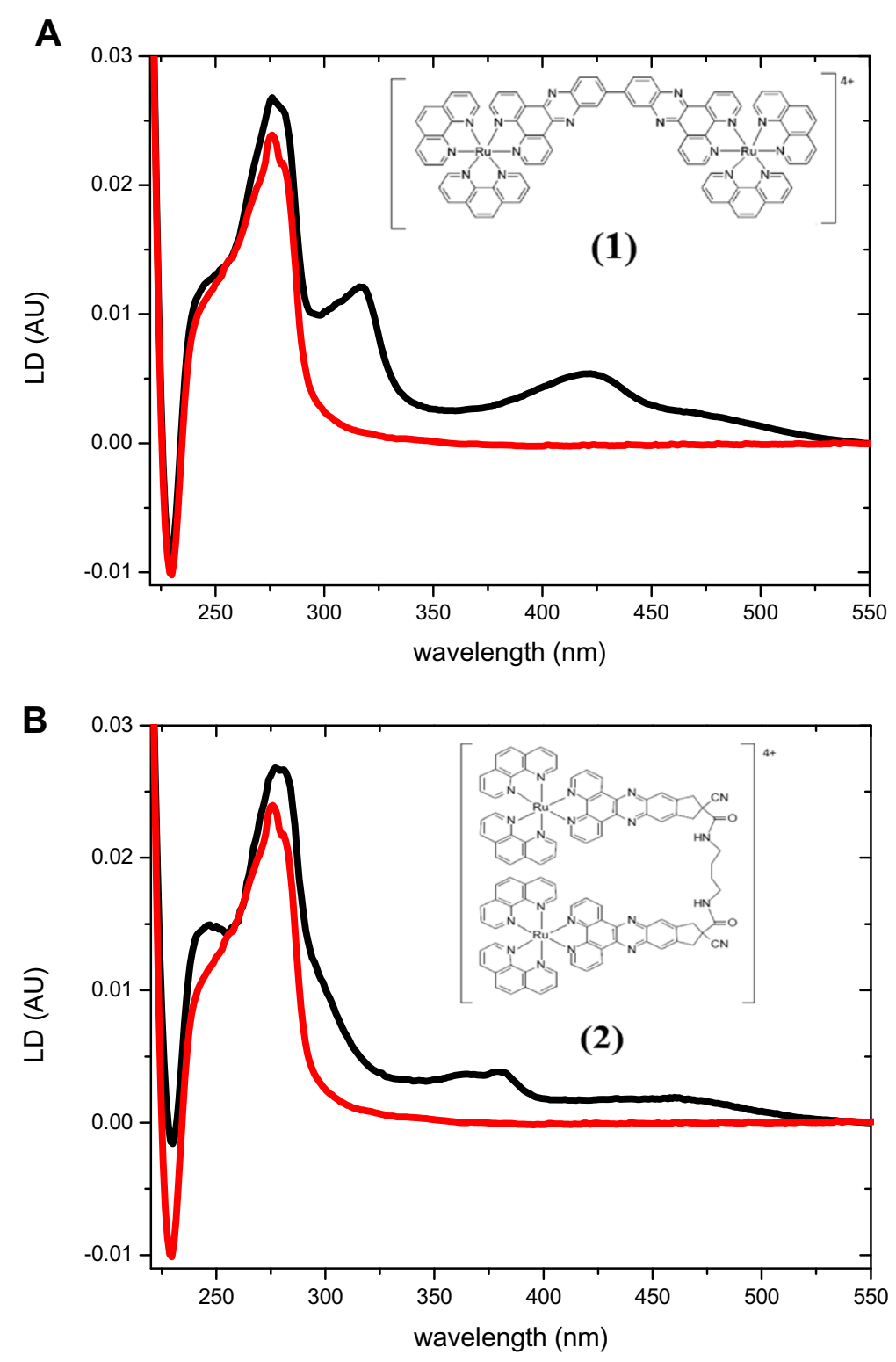

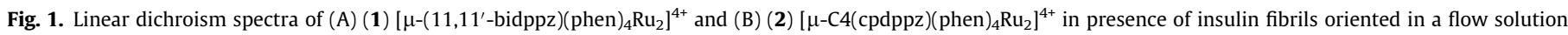

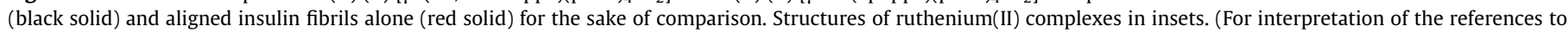
colour in this figure legend, the reader is referred to the web version of this article.)

spectrophotometer equipped with an LD accessory unit, a quartz Couette cell that is used for shear flow orientation of the samples.

Emission was studied using Varian Cary Eclipse spectrofluorometer. The samples were excited at $\lambda_{\mathrm{ex}}=410 \mathrm{~nm}$ and emission recorded at $\lambda_{\mathrm{em}}=625 \mathrm{~nm}$.

Two-photon excited emission were carried out using a laser system consisting of a Quantronix Integra-C regenerative amplifier operating at $800 \mathrm{~nm}$. This system delivers wavelength tunable pulses of $\sim 130$ fs length and was operated at the repetition rate of $1 \mathrm{kHz}$. Emission from amyloids-ruthenium(II) complexes adducts was collected using an Ocean Optics USB 2000 fiber spectrometer that spectral resolution was around $\Delta \lambda \approx 4 \mathrm{~nm}$. In order to remove scattered excitation light, a low pass color filter was used at the entrance of the fiber.

\section{Results and discussion}

LD is defined as the difference in absorbance between light that is linearly polarized parallel and light that is linearly polarized perpendicular to the macroscopic axis of orientation [13]. It studied case LD showed that both complexes are interacting with insulin fibrils and transition dipole moments are oriented parallel to the long molecular axis with absorption maxima at $425 \mathrm{~nm}$ for (1) and $375 \mathrm{~nm}$ for (2) respectively [Fig. 1]. These results are consistent with computational simulations performed for parental, monomeric ruthenium compound [14].

Since the protofilaments building the fibrils are composed of $\beta$-strands oriented perpendicular to the long axis it creates a net with channels that run along the sheet perpendicular to the strands. The $\beta$-sheets in turn form lamella where a separation distance between the sheets is around $1 \mathrm{~nm}$ [15]. Such structural features enables few binding modes of interaction between binuclear ruthenium(II) compounds and fibrils i.e. (a) binding in channels, (b) between laminated sheets (c) in channels between laminated sheets and (d) between the strands. (a), (b) and (c) would lead to parallel alignment of ruthenium(II) complexes and insertion between peptide strands whereas (d) would turn the transition dipole moments into perpendicular position with respect to fibrils 
orientation. The LD results ruled out interaction in mode (d) of both complexes since no rearrangement in binding geometry due to insertion between peptide strands was observed. Also binding in channels between laminated sheets (c) can be excluded due to steric hindrance and the fact that ruthenium complexes are bulky and too large to reach channels between laminated sheets. Most plausible is then binding in channels (a) on the amyloid surface. However there is a large difference in emission intensity between rigid (1) and flexible (2) chromophore upon addition of fibrils which indicates that rate of interaction is related directly to dimers structure [Fig. 2A]. Rigid complex is stiff and cannot bend whereas the one with aliphatic linker have some degree of freedom and thus possibility to adjust between laminated sheets and better protect nitrogen from hydrophilic solvent environment that quench emission [16].
Relying on fluorescence and LD date one can suggest that aliphatic linker of complex (2) can slide between laminated $\beta$-sheets (b) from the channels at the amyloid surface because the separation distance between the sheets is around $1 \mathrm{~nm}$ and this is enough space for aliphatic chain to slide in [Fig. 2B]. In that case the phenanthroline groups bend and LD becomes suppressed as compared to the rigid complex (1). However bending and some rate of flexibility lead to local environment changes around dppz moieties and sliding of linking chain between laminated $\beta$-sheets pulls nitrogen atoms closer to internal structure of the amyloid which is more hydrophobic and what improves the emission efficiency in consequence [Fig 2A]. Intensity in one-photon process is not that important as in case when sample is excited by two-photons. The rigidity leads to $\pi$-conjugation and it is a prerequisite in when dealing with multiphoton spectroscopy or microscopy [17] but in presented

\section{A}

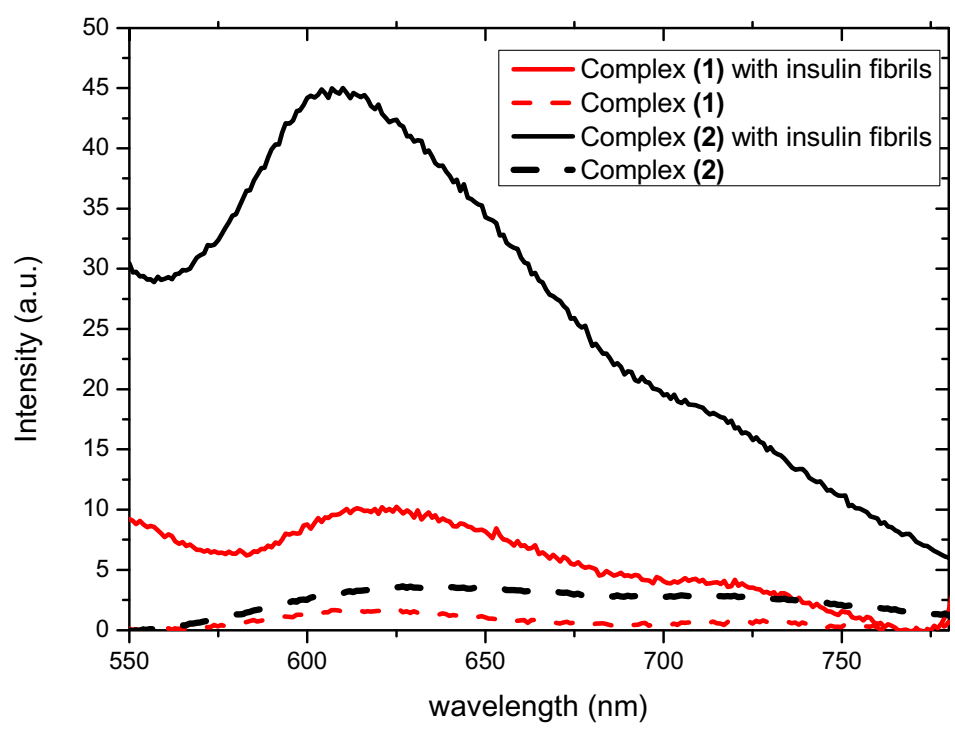

B $\quad$ complex (1)

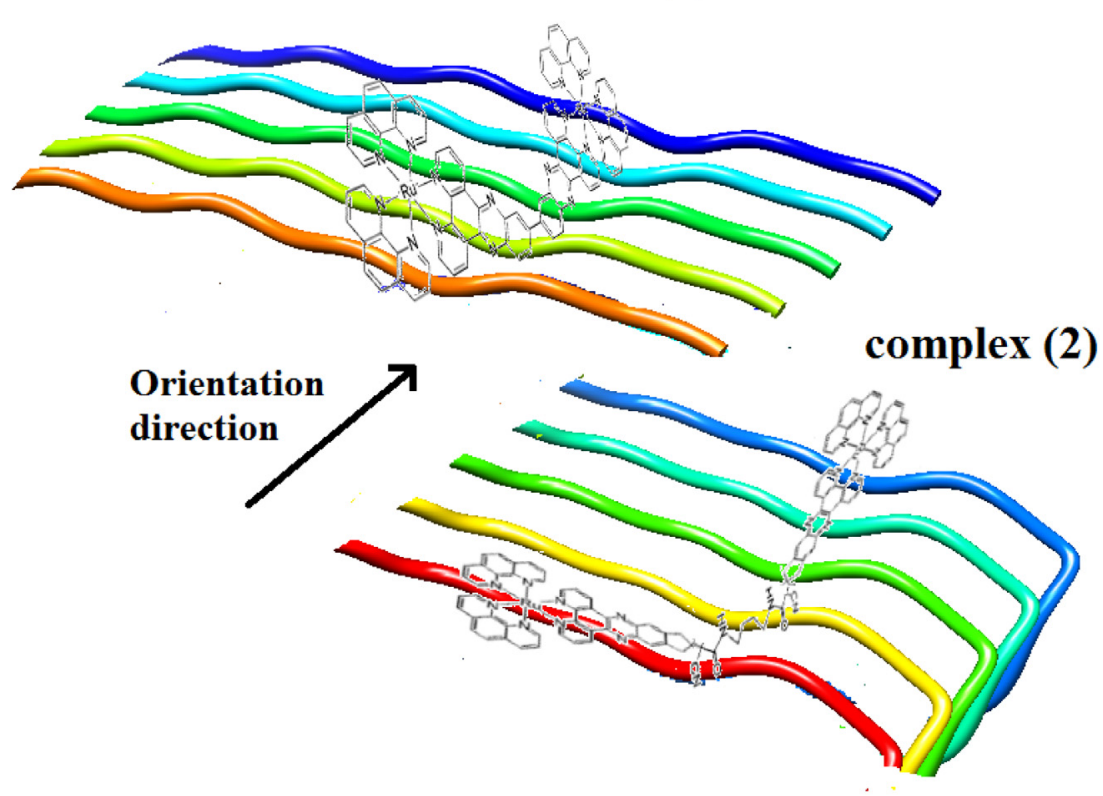

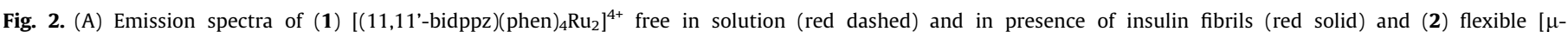

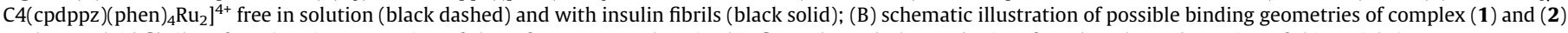
at the amyloid fibril surface. (For interpretation of the references to colour in this figure legend, the reader is referred to the web version of this article.) 


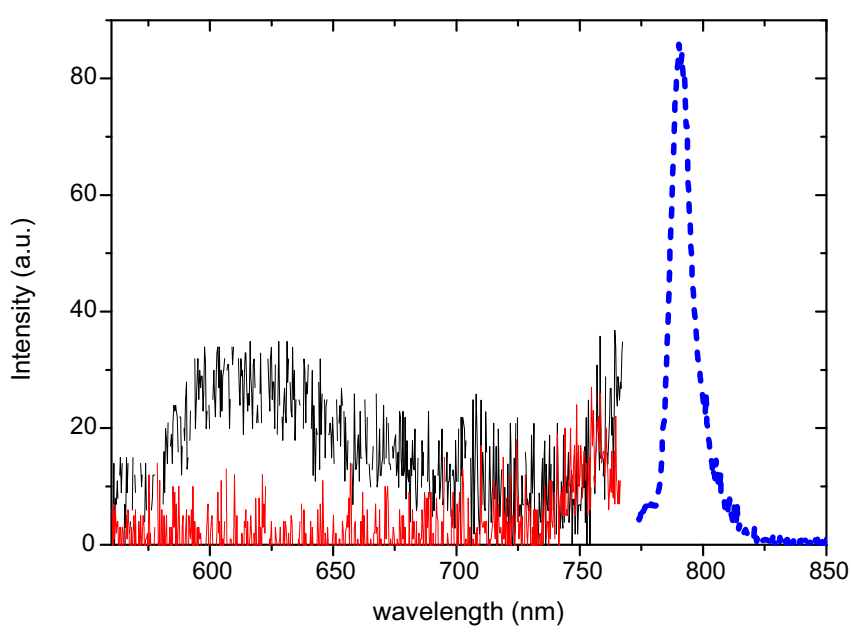

Fig. 3. Two-photon excited emission of complex (2) $\left[\mu-C 4(\operatorname{cpdppz})(\mathrm{phen}){ }_{4} \mathrm{Ru}_{2}\right]^{4+}$ bound to insulin fibrils (black solid) and no emission from complex (1) $\left[\mu-\left(11,11^{\prime}-\right.\right.$ bidppz)(phen) $\left.)_{4} \mathrm{Ru}_{2}\right]^{4+}$ with insulin fibrils (red solid) as a reference. Excitation at $800 \mathrm{~nm}$, peak height normalized (blue dashed). (For interpretation of the references to colour in this figure legend, the reader is referred to the web version of this article.)

case weak emission was observed in presence of amyloids for rigid dimer (1) due to binding geometry that led to strong quenching. Presented results indicate that even though ruthenium(II) complexes are binding to amyloid fibrils there must be additional factors taken into consideration such as complex geometry and flexibility that will permit successful use of metal-organic compounds for imaging applications. Ruthenium(II) complexes as recognition agents for fibrils shall exhibit decent fluorescence properties in order to be detectable in two-photon excitation process which is a nonlinear effect, few orders weaker than a one-photon process. Compound (2) with flexible linker showed moderate emission properties upon exciting the sample with the two-photons by using femtosecond pulses in near-infrared region at $800 \mathrm{~nm}$ [Fig. 3]. Relatively large two-photon absorption (TPA) cross-sections ( $100-150 \mathrm{GM}$ ) of ruthenium(II) complexes in optically transparent window for living organisms 750-1000 nm [17] make them an attractive group for further development in context of detecting amyloid fibrils by advanced multiphoton techniques since commonly used biomarkers such as ethidium bromide or a homodimer [18] as well as amyloid specific chromophores ( 30 GM for dimethylamino substituted GFP-type chromophore (DMC) [19]) are showing few times lower TPA cross-sections.

\section{Conclusions}

In conclusion it is the first report showing that dimer ruthenium(II) complexes can be successfully used for recognition of amyloid protein fibrils what was confirmed by linear dichroism and fluorescence experiments. Binding geometry of the complex determines the fluorescence efficiency and in consequence accessibility for nonlinear optical detection methodology.

\section{Conflict of interest}

There is no conflict of interest.

\section{Acknowledgments}

This work was supported by Swedish Research Council (VR) grant. P.H. thanks Per Lincoln for the gift of ruthenium(II) compounds.

\section{References}

[1] P. Hanczyc, M. Samoc, B. Norden, Nat. Photonics 7 (12) (2013) 969

[2] E. Dawkins, D.H. Small, J. Neurochem. 129 (5) (2014) 756.

[3] D.J. Irwin, V.M.-Y. Lee, J.O. Trojanowski, Nat. Rev. Neurol. 14 (9) (2013) 626.

[4] A. Jacquin, V. Deramecourt, S. Bakchine, C.-A. Maurage, F. Pasquier, J. Neurol 261 (4) (2014) 696.

[5] J. Duboisset, P. Ferrand, W. He, X. Wang, H. Rigneault, S. Brasselet, J. Phys, Chem. B 117 (3) (2013) 784.

[6] P. Hanczyc, P. Lincoln, B. Norden, J. Phys. Chem. B 117 (10) (2013) 2947.

[7] V. Sathisha, E. Babua, A. Ramdass, Z.-Z. Lu, M. Velayudham, P. Thanasekaranb, K.-L. Lub, S. Rajagopal, Talanta 130 (12) (2014) 274.

[8] N.P. Cook, V. Torres, D. Jain, A.A. Martí, J. Am. Chem. Soc. 133 (29) (2011) 11121.

[9] E.J.C. Olson, D. Hu, A. Hormann, A.M. Jonkman, M.R. Arkin, E.D.A. Stemp, J.K. Barton, P.F. Barbara, J. Am. Chem. Soc. 119 (47) (1997) 11458.

[10] E. Baggaley, M.R. Gill, N.H. Green, D. Turton, I.V. Sazanovich, S.W. Botchway, C. Smythe, J.W. Haycock, J.A. Weinstein, J.A. Thomas, Angew. Chem. Int. Ed. 53 (13) (2014) 3435.

[11] M.J. Levene, D.A. Dombeck, K.A. Kasischke, R.P. Molloy, W.W. Webb, J. Neurophys. 91 (4) (2004) 1908.

[12] J. Wigenius, M.R. Andersson, E.K. Esbjörner, F. Westerlund, Biochem. Biophys. Res. Commun. 408 (1) (2011) 115.

[13] B. Norden, A. Rodger, T. Dafforn, Linear Dichroism and Circular Dichroism: A Textbook on Polarized-Light Spectroscopy, Oxford University Press, 2010.

[14] N.P. Cook, M. Ozbil, C. Katsampes, R. Prabhakar, A.A. Martí, J. Am. Chem. Soc. 135 (29) (2013) 10810.

[15] F.G. Bäcklund, J. Wigenius, F. Westerlund, O. Inganäs, N. Solin, J. Mater. Chem. C 2 (37) (2014) 7811.

[16] J. Olofsson, M.L. Wilhelmsson, P. Lincoln, J. Am. Chem. Soc. 126 (47) (2004) 15458.

[17] P. Hanczyc, B. Norden, M. Samoc, Dalton Trans. 41 (11) (2012) 3123.

[18] J. Olesiak-Banska, P. Hanczyc, K. Matczyszyn, B. Norden, M. Samoc, Chem. Phys. 404 (8) (2012) 33.

[19] T.B. Clark, M. Ziółkowski, G.C. Schatz, T. Goodson III, J. Phys. Chem. B 118 (9) (2014) 2351. 